\section{Alternativa de restauración estética en caso de discromía}

Aesthetic restoration alternative in the event of dyschromia. Clinical case

\section{Resumen}

La restauración de un diente tratado endondónticamente con cambio de color representa un reto en la odontología actual, ya que en algunos casos no basta solo con realizar un procedimiento que a nuestro parecer sea el adecuado, sino se puede considerar elegir un conjunto de ellos para obtener resultados satisfactorios. No es suficiente conocer la forma de las piezas dentarias si éstas no se homogenizan con las demás en todos sus aspectos; por consiguiente el color cobra vital importancia para realizar un buen procedimiento restaurador. Con la aplicación de procedimientos como blanqueamiento interno y externo, así como de resinas compuestas se pueden obtener resultados adecuados devolviéndole al paciente la estética y la función perdida. En el presente artículo se detallan algunos criterios básicos a tomar en cuenta en la resolución de un caso de discromía combinando procedimientos de blanqueamiento y de resinas compuestas; los resultados obtenidos fueron satisfactorios ya que se pudo devolver a la paciente la estética y la función perdida. Se concluye que el uso adecuado de estos materiales así como el conocimiento profundo de las técnicas guían el éxito en la resolución de estos casos.

Palabras clave:. blanqueamiento interno, blanqueamiento externo, resinas compuestas, carillas directas.

\begin{abstract}
:
The restoration of a root canal treated tooth with change of color represents a challenge in the current dentistry, since in some cases it is not enough to perform only one procedure that is the appropriate one in our opinion, but it is possible to consider choosing a set of them to obtain satisfactory results. It is not sufficient to know the forms of the dental pieces if these are not homogenized with the other ones in all their aspects; consequently, the color gains vital importance to perform a good restoration procedure. By applying procedures such as internal and external whitening, as well as composite resins, suitable results can be obtained giving back the patient the aesthetics and function lost. In this article, some basic criteria are detailed to be taken into account in the resolution of a case of dyschromia combining whitening and composite resins procedures; the results obtained were satisfactory since it was possible to provide the patient with the aesthetics and function lost. It is concluded that the appropriate use of these materials as well as the deep knowledge of the techniques lead the success in the resolution of these cases. Key works: Internal whitening, external whitening, composite resins, direct resin veneers
\end{abstract}

\section{César Lamas Lara, ${ }^{1}$ Giselle Angulo de la Vega, ${ }^{2}$}

\footnotetext{
${ }^{1}$ Cirujano Dentista, Alumno del Diplomado de Odontología Restauradora y Estética de la Facultad de Odontología de la UNMSM,

Cirujano Dentista, Alumna de la Especialidad de Rehabilitación Oral de la Facultad de Odontología de la UNMSM.
}

\section{Correspondencia:}

CD César Lamas Lara

Facultad de Odontología. UNMSM. Av. Germán Amézaga s/n. Lima 1. Perú

Teléfono: 9994-91403

E-mail: cesar2579@hotmail.com

Fecha de recepción: 14-06-10

Fecha de aprobación: 08-11-10

\section{Introducción}

Las causas de las discromías en dientes tratados endodónticamente pueden ser muy variadas. Restos pulpares que se quedan dentro de la cámara pulpar luego de realizar el tratamiento de conductos, algunos materiales como gutapercha también pueden ocasionar tinciones, así como la difusión de los componentes sanguíneos dentro de los túbulos dentinarios, se consideran como los principales factores de las discromías. ${ }^{1 \text { y } 2}$

La activación de los geles para clareamiento dental con luz halógena, LED o láser, se ha popularizado mucho en estos tiempos, pero no se ha demostrado que los geles tengan algún fotoiniciador que al ser fotoactivados generen una mejor efecto del peróxido en el clareamiento dental. Estudios comparativos demuestran que se obtienen similares resultados fotopolimerizando los geles así como dejándolos actuar sin la necesidad del la activación por luz. ${ }^{3-5}$
La aplicación de técnicas de blanqueamiento realizado en el consultorio o aplicado en cubetas realizados correctamente no afecta la integridad de las piezas dentarias tratadas así como no es un factor desencadenante para la aparición de lesiones de caries. ${ }^{6-8}$

El tiempo de contacto del agente clareador sobre la superficie dentaria y su concentración son factores muy importantes a tomar en cuenta para el éxito de nuestro tratamiento. ${ }^{9}$

En un blanqueamiento interno es muy importante la colocación de un tampón a nivel radicular que llegue a la unión cemento esmalte para prevenir la reabsorción radicular que puede ser producida por la acción del peróxido a ese nivel. ${ }^{10}$

El realizar un clareamiento dental externo e interno en un diente tratado endodónticamente a veces es insuficiente para obtener resultados satisfactorios. El complementar técnicas de clareamiento con restauraciones de resina compuesta nos puede ayudar a optimizar nuestros resultados.

Luego de realizar el procedimiento de clareamiento dental podemos realizar restauraciones con resinas compuestas, esperando un tiempo prudencial para que el efecto de oxígeno residual liberado en el blanqueamiento no afecte nuestro proceso de adhesión. ${ }^{11-13}$

Las resinas compuestas aplicadas en el sector anterior nos ofrecen una alternativa restauradora más, ya que presentan muy buenas propiedades tanto estéticas como mecánicas.

La planificación correcta en estos tipos de casos combinando diferentes procedimientos nos van a ayudar a resolver este tipo de situaciones y de esta manera obtener resultados satisfactorios.

\section{Caso Clínico}

Paciente sexo femenino de 30 años de edad que acude a la Clínica de la Facultad de Odontología de la Universidad 
Nacional Mayor de San Marcos presentando una pieza con cambio de coloración sector anterior preocupada por su problema estético.

$\mathrm{Al}$ examinar el sector ántero superior se puede apreciar la pieza 1.2 con cambio de coloración (discromía) y con una restauración con resina compuesta a nivel mesial.

\section{Diagnóstico}

Del estado de salud general: paciente con buen estado de salud general, sin riesgo sistémico al tratamiento estomatológico.

Del estado de salud estomatológico: paciente con pieza 1.2 discrómica.

\section{Plan de tratamiento}

Se realizó la toma de una radiografía periapical de la pieza 1.2 observando una imagen compatible con material de obturación endodóntico en buen estado. Se realiza la exploración clínica de la pieza 1.2 no apreciando ningún impedimento para realizar algún procedimiento odontológico. Por lo que se planifica realizar para la pieza 1.2 un blanqueamiento interno y externo (técnica mixta), un poste de fibra de vidrio y la confección de una carilla de resina directa.

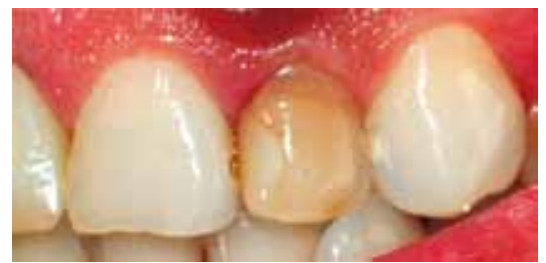

Fig. 1. Situación Inicial.

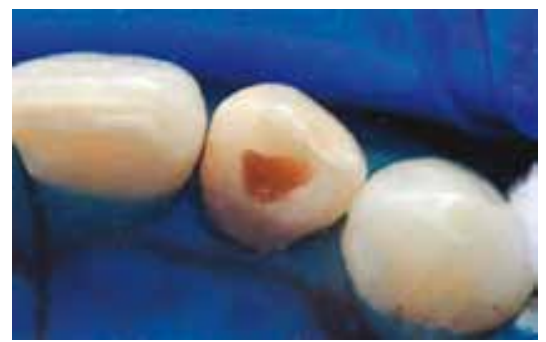

Fig. 2. Se realizó la desobturación del conducto radicular y el posterior sellado con hidróxido de calcio y ionómero de vidrio (tampón)

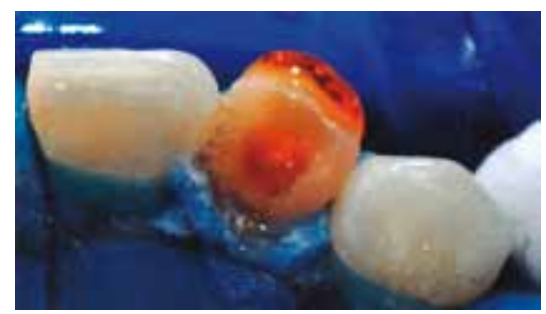

Fig. 3. Se aplicó el gel de peróxido de hidrógeno al $35 \%$ tanto por la cara vestibular como por dentro de la cámara pulpar.

\section{Procedimiento clínico}

Se procedió a realizar la desobturación del conducto radicular ( $4 \mathrm{~mm}$ a partir de cuello anatómico del diente) para la confección de un tampón biológico; se colocó $1 \mathrm{~mm}$ de hidróxido de calcio en pasta en la parte más profunda y todo lo demás fue llenado con ionómero de vidrio modificado con resina hasta el cuello anatómico del diente ( Fig 2).

Se realizó el asilamiento absoluto y se aplicó un gel a base de peróxido de hidrógeno al $35 \%$ tanto por la cara vestibular como dentro de la cámara pulpar (Fig. 3) dejándolo actuar por $30 \mathrm{~min}$ repitiendo las aplicaciones 3 veces por cita.

Una vez terminado las aplicaciones del gel de peróxido de hidrógeno se colocó dentro de la cámara pulpar peróxido de carbamida al $37 \%$ con una torunda de algodón (Fig.4). Se selló la cámara pulpar con ionómero de vidrio modificado con resina (Fig. 5) y se citó a la paciente luego de 7 días para realizar el mismo procedimiento.

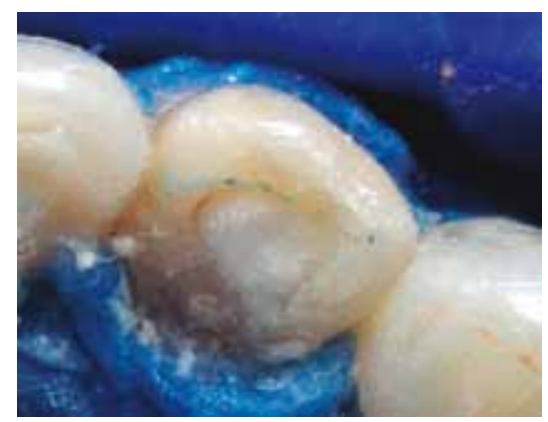

Fig. 4. Se colocó peróxido de carbamida al $37 \%$ dentro de la cámara pulpar con una torunda de algodón.

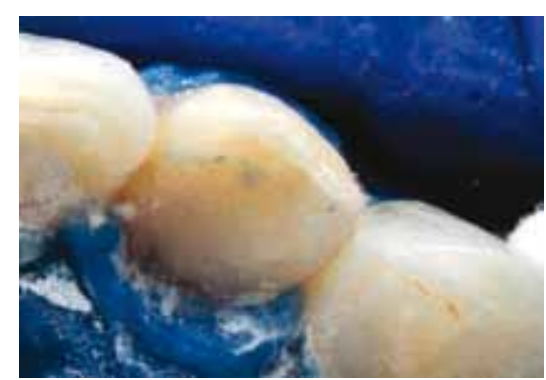

Fig. 5. Se selló la cámara pulpar con iomómero de vidrio y se deja actuar el gel por 7 días.

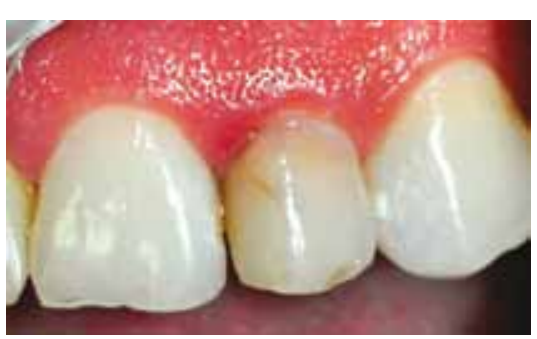

Fig. 6. Vista postblanqueamiento.

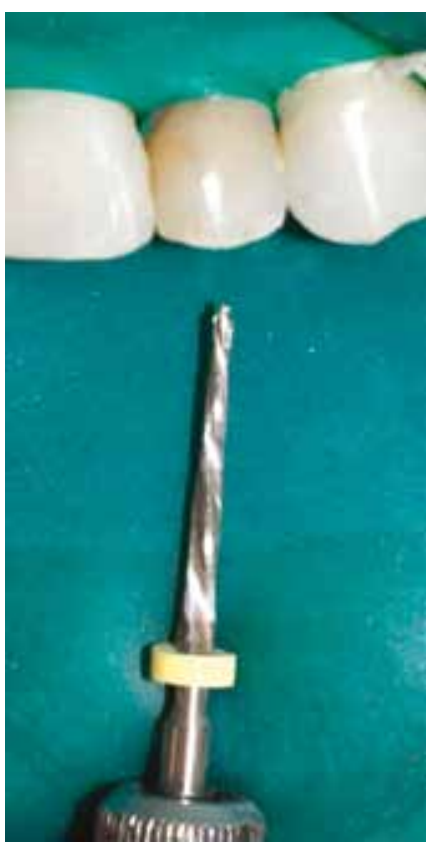

Fig. 7. Desoburación para la colocación de un poste de fibra de vidrio.

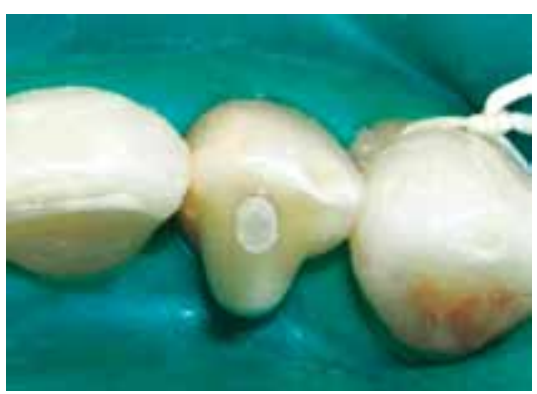

Fig. 8. Cementación del poste de fibra de vidrio con un cemento de resina autograbante y autoacondicionante.

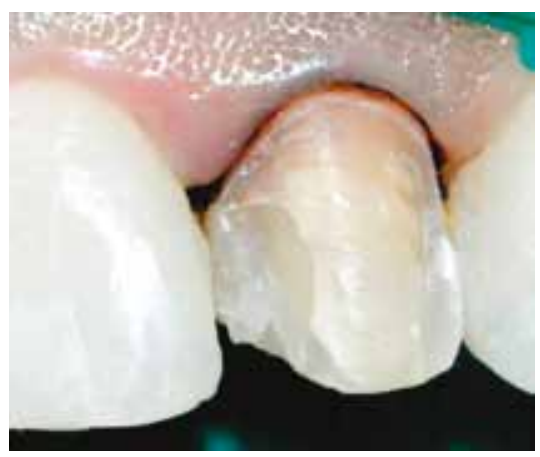

Fig. 9. Se realizó la preparación para la confección de una carilla de resina directa.

Las aplicaciones del gel de peróxido de hidrógeno y de peróxido de carbamida se realizaron por 3 citas. En la última cita al retirar la última torunda de algodón que contiene el peróxido de carbamida al $37 \%$, se limpió la cámara pulpar y se colocó hidróxido de calcio químicamente puro en toda la cámara pulpar, se selló con ionómero de vidrio modificado con resina y se dejó por 2 semanas para neutralizar el peróxido residual que pueda quedar. 
Posteriormente luego de esperar 2 semanas se evaluó el color de la pieza dentaria (Fig. 6). Se realizó la desobturación del conducto radicular dejando $4 \mathrm{~mm}$ de material de obturación a nivel apical para la confección de un poste de fibra de vidrio (Fig. 7). Este poste fue cementado con un cemento de resina autograbante $y$ autoacondicio-

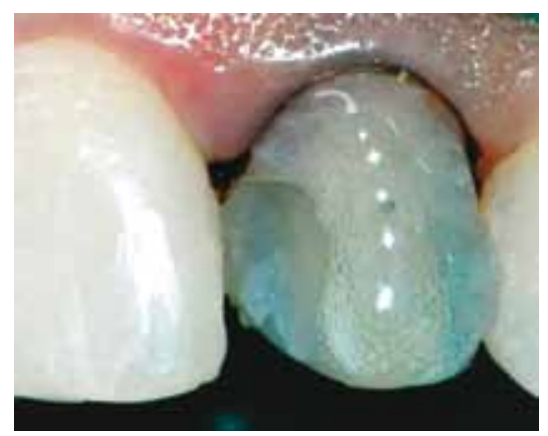

Fig. 10. Grabado con ácido ortofosfórico al 37\%

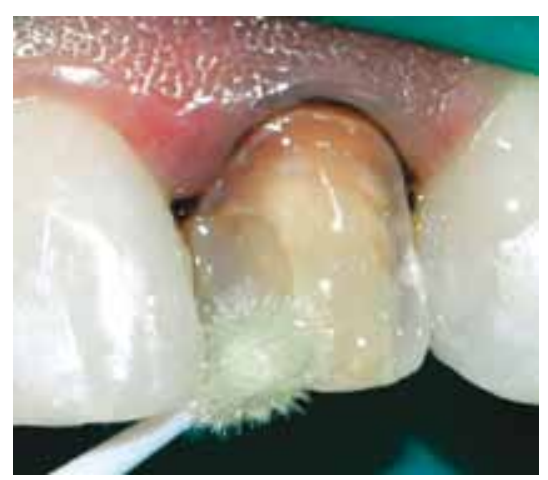

Fig. 11. Aplicación del adhesivo

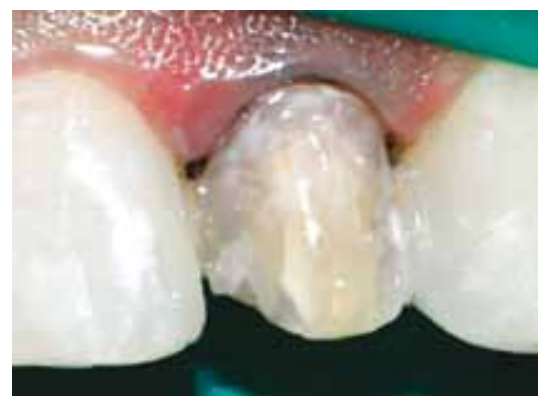

Fig. 12. Aplicación de un tinte blanco para ocultar la dentina oscurecida.

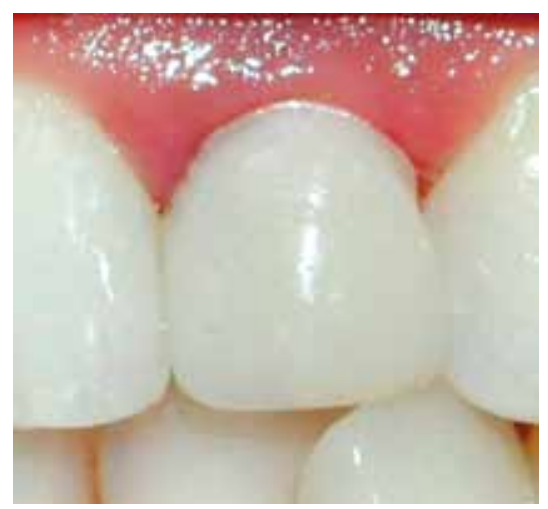

Fig. 13. Restauración terminada nante (Fig. 8). Una vez cementado se retiraron los excesos y se fotopolimerizó por $80 \mathrm{seg}$. Finalmente se colocó resina compuesta para sellar la cámara pulpar.

Posteriormente, se realizó un aislamiento modificado que implica hacer un aislamiento con dique de goma pero en la parte de la pieza a tratar se deja abierto para poder visualizar con mayor eficacia la línea de terminación; y para controlar los fluidos del surco crevicular se colocó hilo de retracción 00 . Se realizó la preparación para una carilla de resina directa (Fig. 9). Se aplicó ácido ortofosfórico sobre la superficie preparada por $30 \mathrm{seg}$. (Fig.10). Se lavó con abundante agua y se secó con papel absorbente. Se aplicó un adhesivo de quinta generación (Fig. 11) y se frotó sobre la superficie hasta evaporar el solvente del adhesivo, se fotopolimerizó por $20 \mathrm{seg}$. Se aplicó un tinte de resina de color blanco (Fig. 12) para ocultar las partes más oscuras de la pieza dentaria y posteriormente se aplicó resina compuesta con la técnica por estratos anatómicos utilizando una resina traslucida como esmalte palatino, un color de cuerpo de blanqueamiento como dentina y un color B1 para el esmalte vestibular (Fig 13). Apreciando una restauración muy similar en color y en forma a las demás piezas dentarias presentes en la arcada.

\section{Resultados}

Los resultados fueron satisfactorios para el paciente ya que se logró devolver la estética y la función a una pieza dentaria con una alteración del color evidente logrando homogenizarla en la arcada dentaria.

\section{Discusión}

Actualmente ha generado mucha controversia el fotoactivar o no los geles blanqueadores. No se ha demostrado que las fuentes luz sea LED, láser, o alguna otra; tenga un efecto real sobre los geles blanqueadores ya que estos geles no contienen en su composición algún fotoiniciador que reaccione con estas fuentes de luz. 3,4 y 5

El realizar un aislamiento modificado para la confección de la carilla de resina compuesta nos permite poder visualizar adecuadamente la línea de terminación de nuestra restauración y controlar adecuadamente los fluidos orales así como la presencia de humedad para que esto no afecte nuestro procedimiento restaurador. ${ }^{13 \text { y } 14}$
El optar por una carilla de resina directa en este caso nos permite realizar un menor desgaste conservando así mayor cantidad de estructura dentaria sana; hoy en día los materiales restauradores directos nos brindan posibilidades estéticas, funcionales y económicas para la resolución de diferentes situaciones clínicas. ${ }^{14}$ y15 Las restauraciones indirectas de cerámica o de alguna resina de laboratorio son opciones validas en estos casos, pero al considerar la edad del paciente se decidió realizar un procedimiento más conservador.

\section{Conclusiones}

Es difícil obtener resultados satisfactorios en casos de piezas dentarias con discromía. El conocer a profundidad los materiales, técnicas actuales así como criterios clínicos para sus aplicaciones cobra vital importancia actualmente.

El conocimiento a cabalidad del matiz, valor y croma de las piezas dentarias, así como las formas van a hacer que nuestras restauraciones presenten mayor naturalidad.

El combinar diferentes técnicas de clareamiento dental puede producir mejores resultados en nuestros tratamientos. Está en el profesional elegir las mejores técnicas para optimizar sus resultados clínicos.

Las resinas compuestas representan una alternativa muy importante actualmente ya que presentan buenas propiedades tanto estéticas como mecánicas.

\section{Referencias bibliográficas}

1. Yui K., Rodrigues J. R., Mancini M., Balducci I., Gonçalves S. Ex vivo evaluation of the effectiveness of bleachingagents on the shade alteration of blood-stained teeth; International Endodontic Journal, 2008;41: 485-492.

2. Attin T., Paque F., Ajam F., Lennon M., Review of the current status of tooth whitening with the walking bleach technique, International Endodontic Journal, 2003;36, 313329.

3. Gomes RS, de Souza FB, Lacerda CM, Bambrilla CFF, Pascotto RC, Avaliação clínica da eficiência do uso do sistema LED-laser, LED e luz halógena na ativação do agente clareador em dentes vitalizados Dental Press Estét, 2008; 5.(2), 62. $-77$ 
4. Carrasco LD, Guerisoli DMZ, Rocha MJA, Pécora JD, Fröner IC Efficacy of intracoronal bleaching techniques with different light activation sources Int Endod J. 2007; 40 (3): 204-8

5. Marson FC, Sensi LG, Vieira LCC, Araújo E. Avaliação clínica do clareamento dental pela técnica no consultório. R Dental Press Estét, 2007;. 4(4): 50-60.

6. Alves EA, Alves FKA, campos EJ, Mathias P. Susceptibility to caries like lesions after dental bleaching with different techniques. Quintessence Int 2007;38(614):404-409.

7. Schiavoni RJ dos S. A micro dureza e a permeabilidad do esmalte humano e bovino. Avaliação após clareamento dental com diferentes técnicas e da aplicação de flúor [dissertaçáo de mestrado] Ribeirão Preto: Ribeirão Preto /USP. Faculdade de Odontologia. Departamento de
Odontologia Restauradora. Opção Endodontia, 2007.

8. Rodrigues JA, Erhardt MCG, Marchi GM, Pimenta LAF, AmbrosanoGMB. Association effect of in-office and nightguard vital bleaching on dental enamel microhardness Braz J Oral Sci.2003; 2(7):365-369.

9. Perdigao J, et al. Ultra morphological study of interaction of dental adhesives with carbamide peroxidebleachede enamel. American Journal of dentistry, Diciembre 1998, No 6.

10. Marin PD. Intra- coronal bleaching: the past, present and future. Ann. Australas Coll Dent Surg. 2006 Sep; 18:36-9.

11. Zhang C, Wang X, Kinoshita J, Zhao B, Toko T, Kimura Y, Matsumoto K. Effects of KTP laser irradiation, diode laser, and LED on tooth bleaching: a comparative study. Photomed Laser Surg. 2007 Apr; 25(2):91-5.

12. Gökay O, Ziramant F, Cali Asal A, Saka OM. Radicular peroxide penetration form carbamide peroxide gels during intracoronal bleaching. Int Endond J. 2008 Marzo 16.

13. Golstein RE, Changing your smile, ed 3. Quintessence Publishers. Chicago 1997.

14. Nogueira de Sá MP, Pascotto RC. Faceta direta em resina composta com recurso de uma matriz de acetato - relato de caso clínico. Revista dental press de estética. 2004; 1:101-111.

15. Bloom DR, Padayachy J N. Smile lifts - a functional and aesthetic perspective. Br Dent J 2006; 200: 199-203. 